This item was submitted to Loughborough's Institutional Repository (https://dspace.lboro.ac.uk/) by the author and is made available under the following Creative Commons Licence conditions.

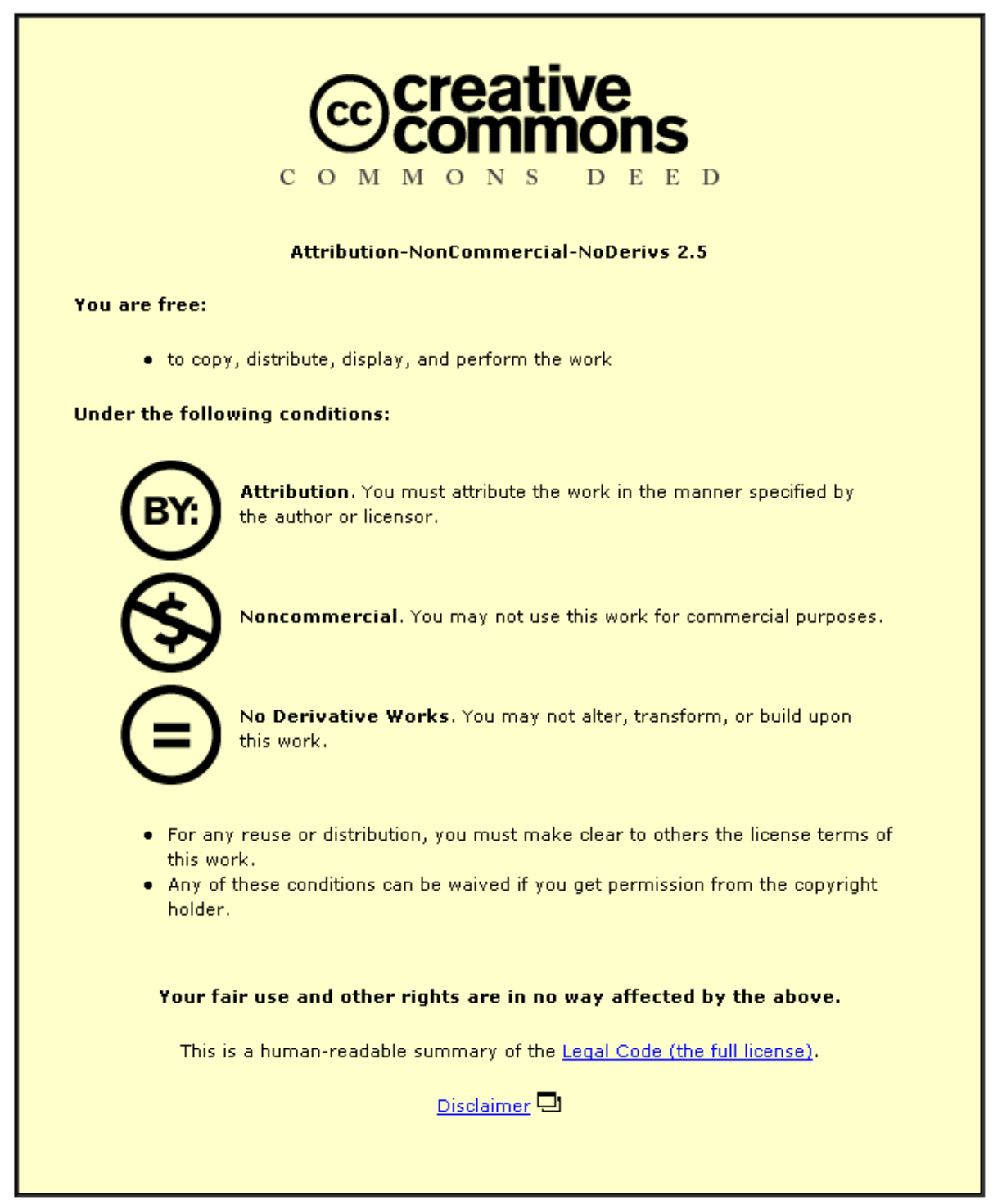

For the full text of this licence, please go to: http://creativecommons.org/licenses/by-nc-nd/2.5/ 


\title{
High temperature superconductivity from realistic Coulomb and Fröhlich interactions
}

\author{
A. S. Alexandrov ${ }^{1,2}{ }^{*}$ J. H. Samson ${ }^{2}{ }^{\dagger}$ and G. Sica ${ }^{2,3}$ \\ ${ }^{1}$ Instituto de Fisica "Gleb Wataghin", Universidade Estadual de Campinas, \\ UNICAMP 13083-970, Campinas, São Paulo, Brasil \\ ${ }^{2}$ Department of Physics, Loughborough University, Loughborough LE11 3TU, UK, EU \\ 3 Dipartimento di Fisica "E.R. Caianiello", Università degli Studi di Salerno, I-84084 Fisciano (SA), Italy, EU
}

\begin{abstract}
In recent years ample experimental evidence has shown that charge carriers in high-temperature superconductors are strongly correlated but also coupled with lattice vibrations (phonons), signalling that the true origin of high- $\mathrm{T}_{c}$ superconductivity can only be found in a proper combination of Coulomb and electron-phonon interactions. On this basis, we propose and study a model for high- $\mathrm{T}_{c}$ superconductivity which accounts for realistic Coulomb repulsion, strong electron-phonon (Fröhlich) interaction and residual on-site (Hubbard $\tilde{U}$ ) correlations without any ad-hoc assumptions on their relative strength and interaction range. In the framework of this model, which exhibits a phase transition to a superconducting state with a critical temperature $\mathrm{T}_{c}$ well in excess of $100 \mathrm{~K}$, we emphasize the role of $\tilde{U}$ as the driving parameter for a BEC/BCS crossover. Our model lays a microscopic foundation for the polaron-bipolaron theory of superconductivity. We argue that the high- $\mathrm{T}_{c}$ phenomenon originates in competing Coulomb and Fröhlich interactions beyond the conventional BCS description.
\end{abstract}

PACS numbers: 74.20.-z, 74.72.-h, 71.38.-k

\section{INTRODUCTION}

Unconventional symmetries of the order parameter allowed some researchers to maintain that a purely repulsive interaction between electrons (Hubbard $U$ ) accounts for superconductivity without phonons in a number of high-temperature superconductors [1]. However, recent analytical [2] and numerical (Monte-Carlo) [3, 4] studies shed doubts on the possibility of high temperature superconductivity from repulsive interactions only.

Also, a growing number of experimental and theoretical results suggest that strong electron correlations and significant electron-phonon interaction (EPI) are the unavoidable features for a microscopic theory of high $\mathrm{T}_{c}$ superconductivity 5 and 6 . In particular, the dopingdependent oxygen-isotope effects on the critical temperature $T_{c}$ and on the in-plane supercarrier mass (ref. [712]), provide direct evidence for a significant EPI and bipolaronic carriers [13] in high-temperature cuprate superconductors. Angle-resolved photoemission spectra (ARPES) [14, 15] provide further evidence for a strong EPI apparently with c-axis-polarised optical phonons [16]. Some theoretical models show that detailed understanding of ARPES requires EPI [15, 17-20] and the lattice disorder [21, 22] to be taken into account along with strong correlations. These results as well as neutron scattering [23, 24], tunnelling [25-27], pump-probe [28], earlier [29] and more recent [30] optical spectroscopies unambiguously show that lattice vibrations play a significant but unconventional role in high-temperature super-

\footnotetext{
* deceased

† Email: j.h.samson@lboro.ac.uk
}

conductivity.

Since first proposed [31], much attention has been paid to the strong EPI as a mechanism of superconductivity providing effective on-site and inter-site attractions between small polarons (electrons dressed by a cloud of phonons) [32]. In the framework of negative Hubbard$U$ and extended negative Hubbard-U models, the strong electron-phonon coupling results in a bound state of two polarons that condense with a Bose-Einstein critical temperature strictly related to the mobility of the pairs [33]. However, the failure of these models in predicting a high critical temperature, due to localization of pairs in the strong coupling regime with some particular (Holstein) EPI, led to a better understanding of more realistic EPIs as the competing interactions with respect to Coulomb repulsion.

Analytical and numerical calculations [34, 35] clarified that EPI with high-frequency optical phonons in ionic solids remains poorly screened signalling the presence of long-range (Fröhlich) EPI at any density of polarons with a remarkable reduction of the polaron effective mass. Consistently, studies on the so called "Fröhlich-Coulomb" model (FCM) $[36,37]$, in which strong long-range EPI and long-range Coulomb repulsion are treated on equal footing, predict light polarons and bipolarons (bound state of two polarons) in cuprates with a remarkably high superconducting critical temperature in the range in which all the interactions are strong compared with the kinetic energy of carriers. The interpretation of the optical spectra of high- $\mathrm{T}_{c}$ materials as the polaron absorption [5, 29, 38] strengthens the view [36] that the Fröhlich EPI is important in those compounds.

In most analytical and numerical models of hightemperature superconductivity proposed so far, one or both of Coulomb and electron-phonon interactions have 
been introduced as input parameters not directly related to the material. Different from those studies an analytical multi-polaron model of high-temperature superconductivity in highly polarisable ionic lattices has been recently proposed [39] and numerically studied (for two-particle states) [40] with generic (bare) Coulomb and Fröhlich interactions avoiding any ad-hoc assumptions on their range and relative magnitude. It has been shown that the generic Hamiltonian comprising any-range Coulomb repulsion and the Fröhlich EPI can be reduced to a shortrange $t-J_{p}$ model at very large lattice dielectric constant, $\epsilon_{0} \rightarrow \infty$, for moderate and strong EPI. In this limit the bare static Coulomb repulsion and EPI negate each other giving rise to a novel physics described by the polaronic $t-J_{p}$ model with a short-range polaronic spin-exchange $J_{p}$ of phononic origin [39].

The cancellation of the bare Coulomb repulsion by the Fröhlich EPI is accurate up to $1 / \epsilon_{0}$ corrections. At finite $\epsilon_{0}$ a residual on-site repulsion of polarons, $\tilde{U}$, could be substantial if the size of the Wannier (atomic) orbitals is small enough. Here we study the effect of this onsite repulsion on the ground state of the extended $t-J_{p}-\tilde{U}$ model accounting for all essential correlations in hightemperature superconductors. It is worth emphasizing that the effect of the on-site $\tilde{U}$ does not follows as a mere generalization of the $t-J_{p}$ model. The residual Hubbard $\tilde{U}$ in fact leads not only to the suppression of on-site pairs but also to the reduction of the exchange interaction and to the Bose-Einstein condensation (BEC) to BCS (BEC/BCS) crossover.

\section{BARE HAMILTONIAN}

Keeping major terms in both interactions, diagonal with respect to sites, yield our generic Hamiltonian in the site representation,

$$
\begin{aligned}
H=- & \sum_{i, j}\left(T_{i j} \delta_{\sigma \sigma^{\prime}}+\mu \delta_{i j}\right) c_{i}^{\dagger} c_{j}+\frac{\alpha}{2 \epsilon_{\infty}} \sum_{i \neq j}^{-} \frac{\hat{n}_{i} \hat{n}_{j}}{|\mathbf{m}-\mathbf{n}|}+ \\
& \sum_{\mathbf{q}, \nu, i} \hbar \omega_{\mathbf{q}} \hat{n}_{i}\left[u_{i}(\mathbf{q}) d_{\mathbf{q}}+H . c .\right]+H_{p h} .
\end{aligned}
$$

Here $T_{i j}$ is the bare hopping integral, if $\mathbf{m} \neq \mathbf{n}$, or the site energy, if $\mathbf{m}=\mathbf{n}, \mu$ is the chemical potential, $i=(\mathbf{m}, \sigma)$ and $j=\left(\mathbf{n}, \sigma^{\prime}\right)$ include both site $(\mathbf{m}, \mathbf{n})$ and $\operatorname{spin}\left(\sigma, \sigma^{\prime}\right)$ quantum numbers, $c_{i}, d_{\mathbf{q}}$ are electron and phonon operators respectively, $\hat{n}_{i}=c_{i}^{\dagger} c_{i}$ is a site occupation operator, $\alpha=e^{2} / 4 \pi \epsilon_{00}\left(\epsilon_{00} \approx 8.85 \times 10^{-12} \mathrm{~F} / \mathrm{m}\right.$ is the vacuum permittivity), and $H_{p h}=\sum_{\mathbf{q}} \hbar \omega_{\mathbf{q}}\left(d_{\mathbf{q}}^{\dagger} d_{\mathbf{q}}+1 / 2\right)$ with the phonon frequency $\omega_{\mathbf{q}}$.

The EPI matrix element is

$$
u_{i}(\mathbf{q})=(2 N)^{-1 / 2} \gamma(\mathbf{q}) \exp (i \mathbf{q} \cdot \mathbf{m})
$$

with the dimensionless EPI coupling, $\gamma(\mathbf{q})(N$ is the number of unit cells). Deriving the generic Hamiltonian in the site representation [39] we approximate the Wannier orbitals as delta-functions, which is justified as long as the characteristic wave-length of doped carriers significantly exceeds the orbital size $a_{0}$. A singular on-site $(\mathbf{m}=\mathbf{n})$ Coulomb repulsion of two carriers with opposite spins (the Hubbard $U$ ) is infinite in this approximation. In fact, it should be cut at $\approx \alpha / \epsilon_{\infty} a_{0}$ as indicated by the bar above the sum, $\sum$. Also for mathematical transparency we consider a single-electron band dropping the electron band index.

Quantitative calculations of the EPI matrix elements in semiconductors and metals have to be performed numerically from pseodopotentials. Fortunately one can parametrize EPI rather than compute it in many physically important cases [41]. EPI in ionic lattices such as the cuprates is dominated by coupling with polar optical phonons. This dipole interaction is much stronger than the deformation potential coupling to acoustic phonons and other multipole EPIs. While the EPI matrix elements are ill-defined in metals, they are well defined in doped insulators, which have their parent dielectric compounds with well-defined phonon frequencies $\omega_{\mathbf{q}}$ and the electron band dispersion.

To parameterize EPI one can calculate the lowest order two-particle vertex function comprising the direct Coulomb repulsion and a phonon exchange [41],

$$
\Gamma\left(\mathbf{q}, \Omega_{n}\right)=\frac{4 \pi \alpha}{\epsilon_{\infty} V_{0} q^{2}}+|\gamma(\mathbf{q})|^{2}\left(\hbar \omega_{\mathbf{q}}\right)^{2} \mathcal{D}\left(\mathbf{q}, \Omega_{n}\right) .
$$

Here $\mathbf{q}=\mathbf{k}_{1}^{\prime}-\mathbf{k}_{1}, \Omega_{n}=\omega_{n^{\prime} 1}-\omega_{n 1}$ are the momentum and energy transfer in a scattering process of two carriers with the initial momenta and the Matsubara frequencies $\mathbf{k}_{1,2}$ and $\omega_{n 1,2}$, respectively, and $\mathcal{D}\left(\mathbf{q}, \Omega_{n}\right)=-\hbar \omega_{\mathbf{q}} /\left[\Omega_{n}^{2}+\right.$ $\left.\left(\hbar \omega_{\mathbf{q}}\right)^{2}\right]$ is the propagator of a phonon of frequency $\omega_{\mathbf{q}}$, and $V_{0}$ is the unit cell volume. In the static limit, $\Omega_{n}=$ 0, Eq.(3) yields the Fourier component of the particleparticle interaction as

$$
\Gamma(\mathbf{q}, 0)=\frac{4 \pi \alpha}{\epsilon_{\infty} V_{0} q^{2}}-|\gamma(\mathbf{q})|^{2} \hbar \omega_{\mathbf{q}}
$$

On the other hand, two static carriers localised on sites $\mathbf{m}$ and $\mathbf{n}$ in the ionic lattice repel each other with the Coulomb potential

$$
v_{i j}=\frac{\alpha}{\epsilon_{0}|\mathbf{m}-\mathbf{n}|},
$$

where the static dielectric constant, $\epsilon_{0}$ accounts for the screening by both core electrons and ions. Comparing Eq.(4) and Eq.(5) we find

$$
|\gamma(\mathbf{q})|^{2} \hbar \omega_{\mathbf{q}}=\frac{\alpha}{\kappa} \sum_{\mathbf{m}}^{-} \frac{e^{i \mathbf{q} \cdot \mathbf{m}}}{m} \approx \frac{4 \pi \alpha}{\kappa V_{0} q^{2}}
$$

at relatively small $q \leq 1 / a$. Here $a$ is the lattice constant and $\kappa=\epsilon_{0} \epsilon_{\infty} /\left(\epsilon_{0}-\epsilon_{\infty}\right)$ with the high-frequency dielectric constant $\epsilon_{\infty}$. The static dielectric constant $\epsilon_{0}$ and the high-frequency dielectric constant $\epsilon_{\infty}$ are readily measured by putting the parent insulator in a capacitor and as the square of the refractive index of the insulator, respectively. Hence, different from many models of 
high-temperature superconductors proposed so far, our generic Hamiltonian with the bare Coulomb and Fröhlich interactions is defined through the measurable material parameters.

\section{III. $t-J_{p}$ AND $t-J_{p}-\tilde{U}$ MODELS}

Using the Lang-Firsov (LF) canonical transformation [42] one can integrate out most of both interactions in the transformed Hamiltonian [39],

$$
\tilde{H}=-\sum_{i, j}\left(\hat{\sigma}_{i j} \delta_{\sigma \sigma^{\prime}}+\tilde{\mu} \delta_{i j}\right) c_{i}^{\dagger} c_{j}+H_{p h}+\frac{1}{2} \sum_{i \neq j} v_{i j} n_{i} n_{j}
$$

since the residual repulsion, $v_{i j}$, is substantially diminished by the large dielectric constant of the polar lattice [see Eq.(5)]. Here

$$
\hat{\sigma}_{i j}=T_{i j} \hat{X}_{i}^{\dagger} \hat{X}_{j}
$$

is the renormalised hopping integral involving multiphonon transitions with $\hat{X}_{i}=\exp \left[\sum_{\mathbf{q}} u_{i}(\mathbf{q}) d_{\mathbf{q}}-H . c.\right]$ and $\tilde{\mu}$ is the chemical potential shifted by the polaron binding energy.

Then, using the Schrieffer-Wolf (SW) canonical transformation [43] and neglecting $v_{i j}$, the transformed Hamiltonian Eq.(7) is reduced to the $t-J_{p}$ Hamiltonian as [39]

$$
\begin{aligned}
\mathcal{H}_{t J_{p}}= & -\sum_{i, j} t_{i j} \delta_{\sigma \sigma^{\prime}} c_{i}^{\dagger} c_{j}+ \\
& 2 \sum_{\mathbf{m} \neq \mathbf{n}} J_{p}(\mathbf{m}-\mathbf{n})\left(\mathbf{S}_{\mathbf{m}} \cdot \mathbf{S}_{\mathbf{n}}+\frac{1}{4} n_{\mathbf{m}} n_{\mathbf{n}}\right) .
\end{aligned}
$$

Here the sum over $\mathbf{n} \neq \mathbf{m}$ counts each pair once only, $\mathbf{S}_{\mathbf{m}}=(1 / 2) \sum_{\sigma, \sigma^{\prime}} c_{\mathbf{m} \sigma}^{\dagger} \vec{\tau}_{\sigma \sigma^{\prime}} c_{\mathbf{m} \sigma^{\prime}}$ is the spin $1 / 2$ operator $\left(\vec{\tau}\right.$ are the Pauli matrices), $n_{\mathbf{m}}=n_{\mathbf{m} \uparrow}+n_{\mathbf{m} \downarrow}$, and $n_{\mathbf{m} \uparrow, \downarrow}=c_{\mathbf{m} \uparrow, \downarrow}^{\dagger} c_{\mathbf{m} \uparrow, \downarrow}$ are site occupation operators.

All quantities in the polaronic $t-J_{p}$ Hamiltonian (9) are defined through the material parameters, in particular the polaron hopping integral, $t_{i j}=T(\mathbf{m}-$ $\mathbf{n}) \exp \left[-g^{2}(\mathbf{m}-\mathbf{n})\right]$ with the polaron band-narrowing exponent

$$
g^{2}(\mathbf{m})=\frac{2 \pi e^{2}}{\kappa \hbar \omega_{0} N V_{0}} \sum_{\mathbf{q}} \frac{1-\cos (\mathbf{q} \cdot \mathbf{m})}{q^{2}}
$$

and

$$
J_{p}(\mathbf{m})=T^{2}(\mathbf{m}) / 2 g^{2}(\mathbf{m}) \hbar \omega_{0},
$$

It has been proposed that the $t-J_{p}$ Hamiltonian, Eq.(9), has a high- $\mathrm{T}_{c}$ superconducting ground state protected from clustering [39]. The polaronic exchange $J_{p}$ is attractive for polarons in the singlet channel and repulsive for polarons in the triplet channel. The origin of this exchange attraction is illustrated in Fig.1.

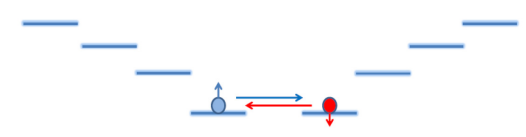

Figure 1. (Colour online) Exchange transfer of two polarons with opposite spins between nearest-neighbour sites with no potential barrier involved. Horizontal lines illustrate atomic levels shifted by the carrier-induced lattice deformation.

If two polarons with opposite spins occupy nearestneighbour sites, they can exchange sites without any potential barrier between them, which lowers their energy by $J_{p}$ proportional to the unrenormalized hopping integral squared.

Importantly the LF transfomation Eq.(7) is exact, and the SW transformation is accurate for intermediate and strong EPI coupling, $\lambda \geq 1 / \sqrt{2 z}$, where $\lambda$ is the BCS coupling constant and $z$ is the lattice coordination number as discussed in details in Ref. 39. The residual repulsion of polarons, $v_{i j}$ in the transformed Hamiltonian, Eq.(7), is small compared with the exchange intersite polaron attraction $J_{p}$ and the short-range bipolaronbipolaron repulsion of about the same magnitude, as long as $\epsilon_{0} \gg \alpha / a J_{p}$. With the typical parameters of the cuprates $J_{p}$ is about $1 \mathrm{eV}$ and $\alpha / a \approx 4 \mathrm{eV}$, so that the residual inter-site repulsion $v_{\mathbf{m n}}$ is small if $\epsilon_{0} \gg 1$, which is well satisfied in all relevant compounds [44].

Nevertheless the on-site term in $v_{\mathbf{m n}}$, Eq.(5), $\tilde{U}$ could be substantial, if the size of the Wannier orbitals is small enough $a_{0} \ll a$. This renormalised $\tilde{U}$ is strongly diminished by the lattice polarization with respect to the bare on-site repulsion. We have emphasised in Refs.39 and 40 that our model describes carriers doped into the charge-transfer Mott-Hubbard (or any polar) insulator, rather than the insulator itself, different from the conventional Hubbard U or t-J models. The bare Hubbard$U$ on the oxygen orbitals (where doped holes reside) in a rigid cuprate lattice is of the same order of magnitude as the on-site attraction induced by the Fröhlich EPI $(\approx 1$ $-2 \mathrm{eV}[44])$, so that the residual Hubbard $\tilde{U}$ could be as large as a few hundred meV. We now take it into account in the energy of a virtual double occupied state $|p\rangle$ with two opposite spins on the same site,

$$
E_{p}-E_{n}=\tilde{U}+\sum_{n_{\mathbf{q}} \neq 0} \hbar \omega_{\mathbf{q}} n_{\mathbf{q}}
$$

Then performing the SW transformation the exchange attraction is found as

$$
J_{p}(u, \mathbf{m}-\mathbf{n})=\frac{t^{2}}{\hbar \omega_{0}} \sum_{k=1}^{\infty} \frac{\left(2 g^{2}(\mathbf{m}-\mathbf{n})\right)^{k}}{k !(k+u)},
$$

where $u=\tilde{U} / \hbar \omega_{0}$. The reduction with respect to $J_{p}(0, \mathbf{m})$ is moderate as long as the relative $u$ is less than $2 g^{2}$, but becomes substantial for $u>2 g^{2}$, Fig. 2 , which puts the characteristic bipolaron binding energy 


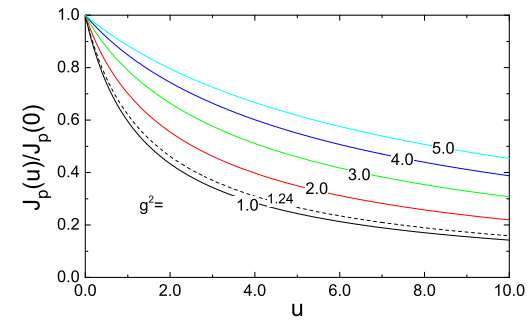

Figure 2. (Colour online) Reduction of the inter-site exchange attraction $J_{p}(u) / J_{p}(0)$ by the on-site residual polaron-polaron repulsion $u=\tilde{U} / \hbar \omega_{0}$ for different values of the polaron mass exponent $g^{2}$.

in the range of a hundred meV comparable with the double pseudogap in the cuprates [5]. Importantly $J_{p}(u)$ remains large or comparable with the polaron hopping integral $t=T(\mathbf{a}) \exp \left[-g^{2}(\mathbf{a})\right]$ since the spin exchange of the $t-J_{p}$ model , Eq.(11), does not contain the small polaron narrowing exponent $\exp \left(-g^{2}\right)$.

Hence our extended $t-J_{p}(u)-\tilde{U}$ model including major correlations effects reads as follows:

$$
\begin{aligned}
\mathcal{H}= & -\sum_{i, j} t_{i j} \delta_{\sigma \sigma^{\prime}} c_{i}^{\dagger} c_{j}+\tilde{U} \sum_{\mathbf{m}} n_{\mathbf{m} \uparrow} n_{\mathbf{m} \downarrow}+ \\
& +2 \sum_{\mathbf{m} \neq \mathbf{n}} J_{p}(u, \mathbf{m}-\mathbf{n})\left(\mathbf{S}_{\mathbf{m}} \cdot \mathbf{S}_{\mathbf{n}}+\frac{1}{4} n_{\mathbf{m}} n_{\mathbf{n}}\right)
\end{aligned}
$$

\section{LOW-DENSITY LIMIT AND HIGH $\mathbf{T}_{c}$}

As in refs 39 and 40 we adopt here the strong-coupling approach to the multi-polaron problem described by the Hamiltonian, Eq.(14), solving first a two-particle problem and then projecting the Hamiltonian on the repulsive Bose gas of small inter-site bipolarons. Such projection allows for a reliable estimate of the superconducting critical temperature for low carrier density as long as bipolarons remain small.

If we neglect the polaronic hopping taking $t=0$, then the ground and the highest energy states are bipolaronic spin-singlet and spin-triplet, respectively, made up of two polarons on neighbouring sites. The zero-energy states [in the nearest-neighbour ( $\mathrm{NN}$ ) approximation] are pairs of polarons separated by more than one lattice spacing. The on-site bipolaron has energy $\tilde{U}>0$.

For $t \neq 0$ our exact diagonalization (ED) results on finite clusters show that the probability to find NN bipolarons falls as we increase the hopping or the strength of the on-site repulsion $\tilde{U}$ as shown in Fig.3 for a $100 \times 100$ square lattice. Consistently, the bipolaron size increases but remains on the order of the lattice spacing in a wide domain of the parameters (see Fig.4). Importantly, although the small bipolaronic configuration persists for any values of the hopping at $\tilde{U}=0$, for $\tilde{U} \neq 0$ and large values of $t$ up to a critical value $t_{c}=\tilde{U} J_{p}(u) /\left(2 \tilde{U}-8 J_{p}(u)\right)$, the presence of a finite on-
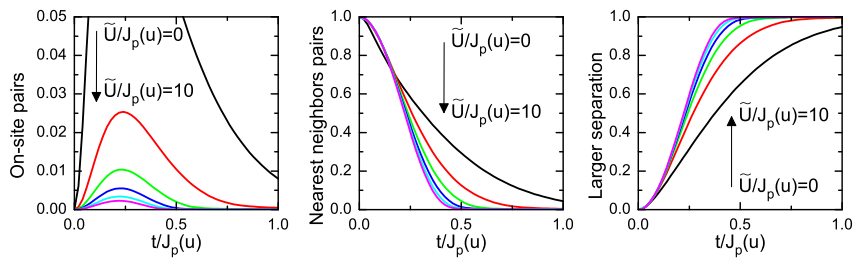

Figure 3. (Colour online) Probability to find two polarons on the same site (left panel), on nearest-neighbour sites $P_{b p}$ (central panel), on more distant sites (right panel) in the ground state of the $t-J_{p}(u)-\tilde{U}$ model on a $100 \times 100$ square lattice with different on-site repulsions $\left(g^{2}=1.24\right)$.
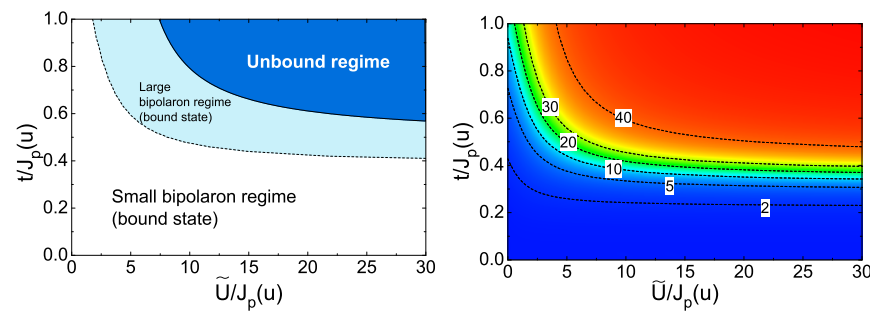

Figure 4. (Colour online) Left panel: phase diagram for the ground state of the polaronic $t-J_{p}(u)-\tilde{U}$ model on a square lattice. Right panel: contourplot of the bipolaron radius $r / a$ ( $a$ is the lattice constant) for a $100 \times 100$ square lattice with periodic boundary conditions. Different numbers represent the value of $r / a$ along the boundaries (dashed lines), emphasizing the increase of the bipolaron radius as we approach the unbound regime. Here $r=\langle|\mathbf{m}-\mathbf{n}|\rangle, \mathbf{m}$ and $\mathbf{n}$ being the position vectors of the two polarons in the bound state.

site interaction leads to the crossover from a small to a large bipolaronic configuration. Finally, for further increasing $t$ the system undergoes a phase transition to an unbound state at $t=t_{c}$. The crossover from a small to a large bipolaronic configuration is also confirmed by the calculation of the bipolaron to polaron effective mass ratio with $m^{* *}=2 m^{*}$ in the large bipolaron regime, as shown in Fig.5.

In the small bipolaron regime, the kinetic energy operator in Eq.(14) connects singlet configurations in the first and higher orders with respect to the polaronic hopping

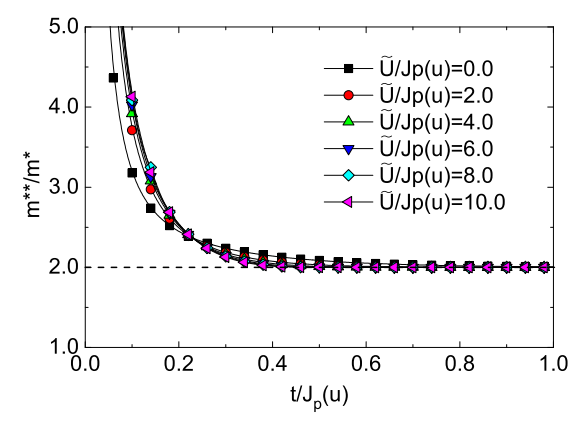

Figure 5. (Colour online) Ratio of bipolaron to polaron mass in the $t-J_{p}(u)-\tilde{U}$ model on a square lattice $\left(g^{2}=1.24\right)$. 


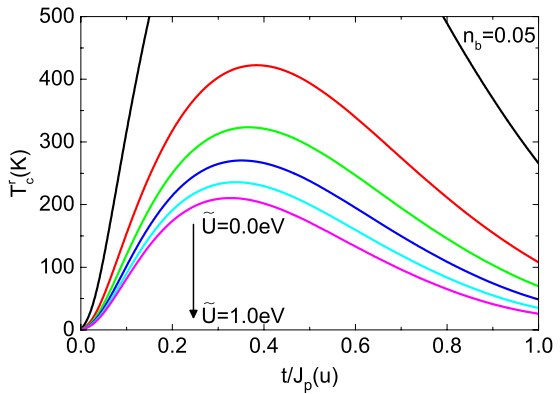

Figure 6. (Colour online) The superconducting critical temperature of the $t-J_{p}(u)-\tilde{U}$ model on the square lattice for low carrier density $n_{b}=0.05 / a^{2}$ with $\hbar \omega_{0}=0.08 \mathrm{eV}, g^{2}=1.24$ and $J_{p}(0)=1.81 \mathrm{eV}$.

integrals. Taking into account only the singlet bipolaron band and discarding all other configurations one can map the $t-J_{p}(u)-\tilde{U}$ Hamiltonian on the hard-core charged Bose gas as described in Ref. 39. This gas is superfluid in $2 \mathrm{D}$ and higher dimensions. In particular, its $2 \mathrm{D}$ critical temperature $T_{c}$ in the dilute limit is given by [45]

$$
T_{c}=\frac{2 \pi \hbar^{2} n_{b}}{k_{B} m^{* *} \ln \ln \left(1 / n_{b} a^{2}\right)},
$$

where $n_{b}$ is the boson density per unit area.

The occurrence of superconductivity in this regime is not controlled by a pairing strength, but by the phase coherence among small bipolarons [31]. At low enough density the Bose-Einstein condensation (BEC) temperature in 3D or its Berezinsky-Kosterlitz-Thouless (BKT) analog in 2D, Eq.(15) should not significantly depend on the bipolaron size as long as it remains small. On the other hand increasing $\tilde{U}$ in our model finally results in a bipolaron overlap, where the bipolaron condensation should appear in the form of the polaronic Cooper pairs in momentum space [46] with a lower critical temperature, rather than in real space (BEC-BCS crossover [46-51]). Hence, we can safely estimate the BEC critical temperature by weighting Eq. 15 with the probability to find $\mathrm{NN}$ polarons as $T_{c}^{r} \approx P_{b p}\left(t / J_{p}(u)\right) T_{c}$ [40].
As shown in Fig.6, for a physical choice of the parameters $\left(\hbar \omega_{0}=0.08 \mathrm{eV}, g^{2}=1.24, T=0.6 \mathrm{eV}\right.$, giving $J_{p}(0)=1.81 \mathrm{eV}$ from Eq. 11) the critical temperature is found to be well in excess of $100 \mathrm{~K}$, despite a low carrier density. Increasing $\tilde{U}$ decreases $J_{p}(u)$, as shown in Fig. 2, and $T_{c}$.

It is worth noting that, unlike in other theories, the strength of the on-site interaction term reduces $T_{c}^{r}$. However, we recall that our residual on site interaction $\tilde{U}$ is defined as the difference between bare Hubbard $U$ and on-site Fröhlich EPI; therefore at $\tilde{U}=0$, when $T_{c}$ is maximized, we have a strong bare on-site interaction with $U \approx 2 E_{p} \sim 1-2 \mathrm{eV}$

\section{CONCLUSIONS}

In conclusion, we have introduced and studied the polaronic $t-J_{p}(u)-\tilde{U}$ model, defined through the bare material parameters. The model, being an essential generalization of the $t$ - $J_{p}$ model [39], includes all electronelectron and electron-phonon correlations providing a microscopic explanation of the high- $\mathrm{T}_{c}$ phenomenon without any ad-hoc approximations. We show that the inclusion of the residual on-site interaction $\tilde{U}$ (neglected in the $t$ - $J_{p}$ model $[39,40]$ ), drives the system to a BEC/BCS crossover that reconciles the polaron-bipolaron theory of superconductivity with the observation of a large Fermi surface in overdoped cuprate superconductors. We offer an explanation, on microscopic grounds, of the high$\mathrm{T}_{c}$ phenomenon as a consequence of competing Coulomb and Fröhlich interactions in highly polarizable ionic lattices beyond the conventional BCS description.

\section{ACKNOWLEDGEMENTS}

We gratefully acknowledge enlightening discussions with Antonio Bianconi, Slaven Barisic, Ivan Bozovic, Victor Kabanov, Ferdinando Mancini, Dragan Mihailovic, Nikolay Plakida, and support from the UNICAMP visiting professorship program and ROBOCON (Campinas, Brasil).
[1] For a comprehensive review see N. Plakida Hightemperature cuprate superconductors: experiment, theory and applications (Springer, Heidelberg 2010).

[2] A. S. Alexandrov and V. V. Kabanov, Phys. Rev. Lett. 106, 136403 (2011).

[3] T. Aimi and M. J. Imada, Phys. Soc. Jpn. 76, 113708 (2007).

[4] T. M. Hardy, J. P. Hague, J. H. Samson, and A. S. Alexandrov, Phys. Rev. B 79, 212501 (2009).

[5] For a recent review see A. S. Alexandrov and J. T. Devreese, Advances in Polaron Physics (Springer, Berlin 2009).
6] A. S. Alexandrov, C. Di Castro, I. Mazin, and D. Mihailovic, Adv. Cond. Matt. Phys. 2010, 206012 (2010), and contributions to this special issue.

[7] G. M. Zhao, and D. E. Morris, Phys. Rev. B 51, R16487 (1995).

[8] G. M. Zhao, K. K. Singh, A. P. B. Sinha, and D. E. Morris, Phys. Rev. B 52, 6840 (1995).

[9] G. M. Zhao, M. B. Hunt, H. Keller, and K. A. Müller, Nature (London) 385, 236 (1997).

[10] G. M. Zhao, K. Konder, H. Keller, and K. A. Müller, J. Phys.: Condens. Matter 10, 9055 (1998).

[11] G. M. Zhao, V. Kirtikar, and D. E. Morris, Phys. Rev. B 63, R220506 (2001). 
[12] R. Khasanov, D. G. Eshchenko, H. Luetkens, E. Morenzoni, T. Prokscha, A. Suter, N. Garifianov, M. Mali, J. Roos, K. Conder, and H. Keller, Phys. Rev. Lett. 92, 057602 (2004).

[13] A. S. Alexandrov and G. M. Zhao, New Journal of Physics 14013046 (2012).

[14] A. Lanzara, P. V. Bogdanov, X. J. Zhou, S. A. Kellar, D. L. Feng, E. D. Lu, T. Yoshida, H. Eisaki, A. Fujimori, K. Kishio, J.-I. Shimoyama, T. Noda, S. Uchida, Z. Hussain, and Z.-X. Shen, Nature (London)412, 510 (2001).

[15] K. M. Shen, F. Ronning, D. H. Lu, W. S. Lee, N. J. C. Ingle, W. Meevasana, F. Baumberger, A. Damascelli, N. P. Armitage, L. L. Miller, Y. Kohsaka, M. Azuma, M. Takano, H. Takagai and Z.-X. Shen, Phys. Rev. Lett. 93, 267002 (2004).

[16] W. Meevasana, N. J. C. Ingle, D. H. Lu, J. R. Shi, F. Baumberger, K. M. Shen, W. S. Lee, T. Cuk, H. Eisaki, T. P. Devereaux, N. Nagaosa, J. Zaanen, and Z.-X. Shen, Phys. Rev. Lett. 96, 157003 (2006).

[17] A. S. Alexandrov and C. J. Dent, Phys. Rev. B 60, 15414 (1999).

[18] A. S. Mishchenko and N. Nagaosa, Phys. Rev. Lett. 93, 036402 (2004).

[19] M. Hohenadler, D. Neuber, W. von der Linden, G. Wellein, J. Loos, and H. Fehske, Phys. Rev. B 71, 245111 (2005).

[20] O. Rosch, O. Gunnarsson, X. J. Zhou, T. Yoshida, T. Sasagawa, A. Fujimori, Z. Hussain, Z.-X. Shen, and S. Uchida, Phys. Rev. Lett. 95, 227002 (2005).

[21] A. S. Alexandrov and K. Reynolds, Phys. Rev. B 76, 132506 (2007)

[22] A. S. Alexandrov, Phys. Rev. B 85, 092501 (2012).

[23] T. R. Sendyka, W. Dmowski, T. Egami, N. Seiji, H. Yamauchi, and S. Tanaka, Phys. Rev. B 51, 6747 (1995).

[24] D. Reznik, L. Pintschovius, M. Ito, S. Iikubo, M. Sato, H. Goka, M. Fujita, K. Yamada, G. D. Gu, and J. M. Tranquada, Nature (London) 440, 1170 (2006).

[25] G. M. Zhao, Phys. Rev. B 75, 214507 (2007).

[26] H. Shim, P. Chaudhari, G. Logvenov, and I. Bozovic, Phys. Rev. Lett. 101, 247004 (2008).

[27] G. M. Zhao, Phys. Rev. Lett. 103, 236403 (2009).

[28] C. Gadermaier et. al, Phys. Rev. Lett. 105, 257001 (2010).

[29] D. Mihailovic, C. M. Foster, K. Voss, and A. J. Heeger, Phys. Rev. B 42, 7989 (1990).
[30] A. S. Mishchenko, N. Nagaosa, Z.-X. Shen, G. De Filippis, V. Cataudella, T. P. Devereaux, C. Bernhard, K. W. Kim, and J. Zaanen, Phys. Rev. Lett. 100, 166401 (2008).

[31] A. S. Alexandrov, J. Ranninger, Phys. Rev. B 23, 1796; Phys. Rev. B 24, 1164 (1981).

[32] A. S. Alexandrov and N. F. Mott, Rep. Prog. Phys. 57, 1197 (1994); Polarons and Bipolarons (World Scientific, Singapore, 1995).

[33] V. M. Loktev, R. M. Quick and S. G. Sharapov, Phys. Rep. 349, 1-123 (2001).

[34] A. S. Alexandrov and A. M. Bratkovsky, Phys. Rev. Lett. 105, 226408 (2010).

[35] T. Bauer and C. Falter, Phys. Rev. B 80, 094525 (2009).

[36] A. S. Alexandrov, Phys. Rev. B 53, 2863 (1996).

[37] A. S. Alexandrov and P. E. Kornilovitch, J. Phys. Condens. Matter 14, 5337 (2002).

[38] J. Tempere and J. T. Devreese, Phys. Rev. B 64104504 (2001).

[39] A. S. Alexandrov, EPL 95, 27004 (2011).

[40] A. S. Alexandrov, J. H. Samson, and G. Sica, Phys. Rev. B 85, 104520 (2012).

[41] G. D. Mahan, Many-Particle Physics (Plenum, New York 1990).

[42] I. G. Lang and Yu. A. Firsov, Zh. Eksp. Teor. Fiz. 43 1843 (1062) [Sov. Phys. JETP 16 , 1301 (1963)].

[43] J. R. Schrieffer and P. A. Wolff, Phys. Rev. 149, 491 (1966).

[44] A. S. Alexandrov and A. M. Bratkovsky, Phys. Rev. Lett. 84, 2043 (2000).

[45] D. S. Fisher and P. C. Hohenberg, Phys. Rev. B 37, 4936 (1988).

[46] A. S. Alexandrov, Russ. J. Phys. Chem. 57, 167 (1983).

[47] D. M. Eagles, Phys. Rev. 186, 456 (1969); Phys. Today 62 (8), 8 (2009).

[48] A. J. Leggett, J. Phys. Colloq. 41, C7 (1980).

[49] P. Nozieres and S. Scmittrink, J. Low Temp. Phys. 59, 195 (1985).

[50] R. Micnas, J. Ranninger, and S. Robaszkiewicz, Rev. Mod. Phys. 62, 113 (1990).

[51] Q. J. Chen, J. Stajic, S. Tan, and K. Levin, Phys. Rep. 412, 1 (2005). 